Proceedings

\title{
Survival Proteins Encourage Human T Helper 17 Cells to Escape from Cell Death ${ }^{\dagger}$
}

\author{
Tuğçe Çimen and Ayten Nalbant* \\ Molecular Immunology Laboratory, Department of Molecular Biology and Genetics, Izmir Institute of \\ Technology, İzmir 35430, Turkey; tugcecimen24@gmail.com \\ * Correspondence: aytennalbant@iyte.edu.tr \\ + Presented at the 2nd International Cell Death Research Congress, Izmir, Turkey, 1-4 November 2018. \\ Published: 6 December 2018
}

\begin{abstract}
IL-17 producing T helper 17 (Th17) cells are identified as a distinct subset of CD4 ${ }^{+} \mathrm{T}$ helper cells. They play a role in immune response. Abnormal regulation of Th17 cells can play a role in different type of pathologies such autoimmune diseases and cancer. The apoptotic and survival mechanisms of Th17 cells are not well known in different type of diseases. Therefore, the aim of the study was to investigate Bcl-2 family proteins to understand the regulation network of apoptosis in human Th17 cells. To do that, Peripheral blood (PB) were drawn from the healthy volunteers. Peripheral Blood Mononuclear Cells (PBMCs) were isolated from blood by Ficoll gradient isolation method. The naïve $\mathrm{CD}^{+} \mathrm{T}$ cells were isolated from PBMC. Sorted naive $\mathrm{CD} 4^{+} \mathrm{T}$ cells were cultured under Th17 polarizing conditions. Th17 cells were characterized by Flow cytometry. Cell lysates were obtained from negative controls and Th17 cells. Bcl-2 family members (Bik, BID and Puma) in Th17 cells were detected by western blot. Data showed that naive T cells were differentiated into Th17 cells. Then, cell lysate of this cells were used for western blot experiments. In the Th17 cell lysate, BH3 family members Bik and Puma were not detectable but Mcl-1 expression was increased. Overall data indicated that the pro-apoptotic BH3-only subgroup proteins Bik and Puma was not detectable, however anti-apoptotic Mcl-1 protein expression was increased.
\end{abstract}

Keywords: Th17 cells; Bik; Puma; survival

\section{Introduction}

$\mathrm{T}$ helper (Th) cells are responsible for the regulation of the adaptive immune response. Newly discovered IL-17A and IL-17F producing T helper 17 (Th17) cells protect the host from bacterial or fungal infection. However, uncontrolled Th17 cell responses are often associated with pathological conditions including autoimmune diseases. For instance, human in vitro studies have showed that there was an impaired apoptotic deletion of polyclonal and myelin specific $T$ cells which were derived from Multiple Sclerosis patients' blood [1]. In the literature, there is a limited amount of data to show the elimination mechanisms of human Th17 effector cells after they carried out their function [2].

Apoptosis is a programmed cell death and it involves in tissue homeostasis, development and unwanted cell elimination in multicellular organisms. Dysregulated apoptosis can play a role in different diseases. There are extrinsic and intrinsic pathways to mediate apoptosis. B-cell-lymphoma-2 (Bcl-2) family proteins are major regulators of intrinsic apoptotic pathway. Bcl-2 family proteins regulates apoptosis by balancing the anti-apoptotic and pro-apoptotic activities [3]. Anti-apoptotic and pro-apoptotic proteins of $\mathrm{Bcl}-2$ family are classified by $\mathrm{Bcl}-2$ homology $(\mathrm{BH})$ domains. $\mathrm{BH}$ domains are called as $\mathrm{BH} 1, \mathrm{BH} 2, \mathrm{BH} 3$ and $\mathrm{BH} 4$. Anti-apoptotic family consists of $\mathrm{BH} 1, \mathrm{BH} 2, \mathrm{BH} 3$ and BH4 domains. It consist of Bcl-2, Bcl-xL, MCL1, Bcl-W, Bcl-B and BCL2A1 proteins. Pro-apoptotic proteins are divided into 2 subgroups. First subgroup pro-apoptotic proteins are BH123 subfamily, 
which lacks BH4 domain and includes BAX, BAK and BOK and interacts with anti-apoptotic proteins. Second subgroup pro-apoptotic proteins are BH3 only subfamily, which lacks BH1, 2 and 4 domains and comprises of BID, BIM, BIK, PUMA, NOXA and BAD [3]. In this study, anti-apoptotic Mcl-1and pro-apoptotic Bik and PUMA in Th17 cells were measured by using western blot method.

\section{Materials and Methods}

Ethics approval for this study was obtained from the Noninvasive Ethics Committee of Dokuz Eylül University, İzmir, Turkey. The venous blood were drawn from the healthy volunteers by health professionals at the Dokuz Eylül University Blood Bank. Peripheral blood mononuclear cells (PBMCs) were isolated from blood by ficoll separation method. Naive CD4 ${ }^{+} \mathrm{T}$ cells from PBMC were sorted by magnetic sorting with Variomacs. Sorted cells were stained via cell surface markers for flow cytometric analysis including PERCP-CD4, FITC-CD45RA, APC-CD45RO and PE-CD69. Samples were acquired by Guava EasyCyte system. Sorted cells were cultured with CD3, CD28, IL-1-beta, IL-6 and TGF-beta pure proteins to differentiate naive $\mathrm{CD}^{+} \mathrm{T}$ cells into Th17 cells. Medium alone is used as negative control. Th17 cells were identified with RORC expression, which is detected by western blot. Cell lysates for western blot were obtained from negative control and Th17 cell cultures at different time points. Samples and molecular weight marker were loaded into gel and runned at $80 \mathrm{~V}$ for $2 \mathrm{~h}$. The transferred membrane was incubated with TBS-T milk solution for $1 \mathrm{~h}$ on the shaker and washed with TBS-T three times for $5 \mathrm{~min}$. To detect molecules on the PVDF membrane, RORC2, Bik, Puma and Mcl-1 antibodies were incubated overnight at $4{ }^{\circ} \mathrm{C}$. Then, HRP linked-secondary antibody was used. Finally, blot was incubated with enhancer solution to visualize protein bands in Fusion FX chemiluminescence system. B-actin is used as positive control for cytoplasmic proteins.

\section{Results}

Data showed that expression of Th17 cell specific cytokines confirmed that cells were Th17 cells. Then, cell lysate of this cells were used for western blot experiments. Bcl-2 family proteins, a specific subdomain that is known as BH3 family which contain Bik, BID and Puma were studied. BH3 family proteins do not include $\mathrm{BH} 1$ or $\mathrm{BH} 2$ domains and they trigger apoptosis by binding to anti-apoptotic $\mathrm{Bcl}-2$ protein family members. Bik is belongs to $\mathrm{BH} 3$ only protein family member that maintains apoptosis by interacting with other anti or pro-apoptotic proteins. If Bik is activated by phosphorylation, it can be detected by western blot. In the Th17 cell lysate, Bik was first BH3 only protein measured. It was not detectable even though there was a good amount of beta-actin as control. PUMA is a pro-apoptotic member of the BH3-only subgroup of the Bcl-2 family and p53 upregulated modulator of apoptosis. PUMA mediates apoptosis by interacting directly with antiapoptotic members. It mainly localizes to the mitochondria. The binding of PUMA to the antiapoptotic members (Bcl-2-like proteins) is a critical regulatory step in apoptosis induction. In the Th17 cell lysate, PUMA was not detectable. In contrast to the pro-apoptotic BH3-only subgroup proteins Bik and Puma, Mcl-1 is anti-apoptotic protein that belongs to the Bcl-2 family. Western blot data showed that the anti-apoptotic protein Mcl-1 expression in the Th17 cells was increased.

\section{Discussion}

Previously, Kryczek et al. showed that human Th17 cells display anti-apoptotic properties. This group focused on hypoxia inducible factor $1 \alpha$ which has crucial role on apoptosis and survival of Th17 cells by controlling gene expression of Bcl-2 family proteins via Notch signaling pathway [4]. Data from our study indicated that the pro-apoptotic BH3-only subgroup proteins Bik and Puma was not detectable, but expression of Mcl-1 was increased in cells incubated under Th17 polarization conditions. Findings of the study suggest that Th17 cells can live longer. Taken together, it seems like survival of Th17 cells can be regulated by different signaling pathways.

Acknowledgments: This work was supported by the Scientific and Technological Research Council of Turkey (TÜBİTAK) (Grant number: 215Z127 to Ayten Nalbant). The funders had no role in study design, data collection, and analysis, decision to publish, or preparation of the manuscript. 


\section{References}

1. Durelli, L.; Conti, L.; Clerico, M.; Boselli, D.; Contessa, G.; Ripellino, P.; Ferrero, B.; Eid, P.; Novelli, F. Thelper 17 cells expand in multiple sclerosis and are inhibited by interferon-beta. Ann. Neurol. 2009, 65, 499-509.

2. Volpe, E.; Sambucci, M.; Battistini, L.; Borsellino, G. Fas-Fas Ligand: Checkpoint of T Cell Functions in Multiple Sclerosis. Front. Immunol. 2016, 7, 382.

3. Chipuk, J.E.; Moldoveanu, T.; Llambi, F.; Parsons, M.J.; Green, D.R. The BCL-2 family reunion. Mol. Cell 2010, 37, 299-310, doi:10.1016/j.molcel.2010.01.025.

4. Kryczek, I.; Zhao, E.; Liu, Y.; Wang, Y.; Vatan, L.; Szeliga, W.; Moyer, J.; Klimczak, A.; Lange, A.; Zou, W. Human TH17 cells are long-lived effector memory cells. Sci. Transl. Med. 2011, 3, 104ra100, doi:10.1126/scitranslmed.3002949.

(C) 2018 by the authors. Licensee MDPI, Basel, Switzerland. This article is an open access article distributed under the terms and conditions of the Creative Commons Attribution (CC BY) license (http://creativecommons.org/licenses/by/4.0/). 